This item was submitted to Loughborough's Research Repository by the author.

Items in Figshare are protected by copyright, with all rights reserved, unless otherwise indicated.

\title{
The Markov network fitness model
}

PLEASE CITE THE PUBLISHED VERSION

http://link.springer.com/chapter/10.1007/978-3-642-28900-2_8

VERSION

AM (Accepted Manuscript)

LICENCE

CC BY-NC-ND 4.0

\section{REPOSITORY RECORD}

Brownlee, Alexander E.I., Siddhartha Shakya, and John McCall. 2019. "The Markov Network Fitness Model". figshare. https://hdl.handle.net/2134/8916. 
This item was submitted to Loughborough's Institutional Repository (https://dspace.lboro.ac.uk/) by the author and is made available under the following Creative Commons Licence conditions.

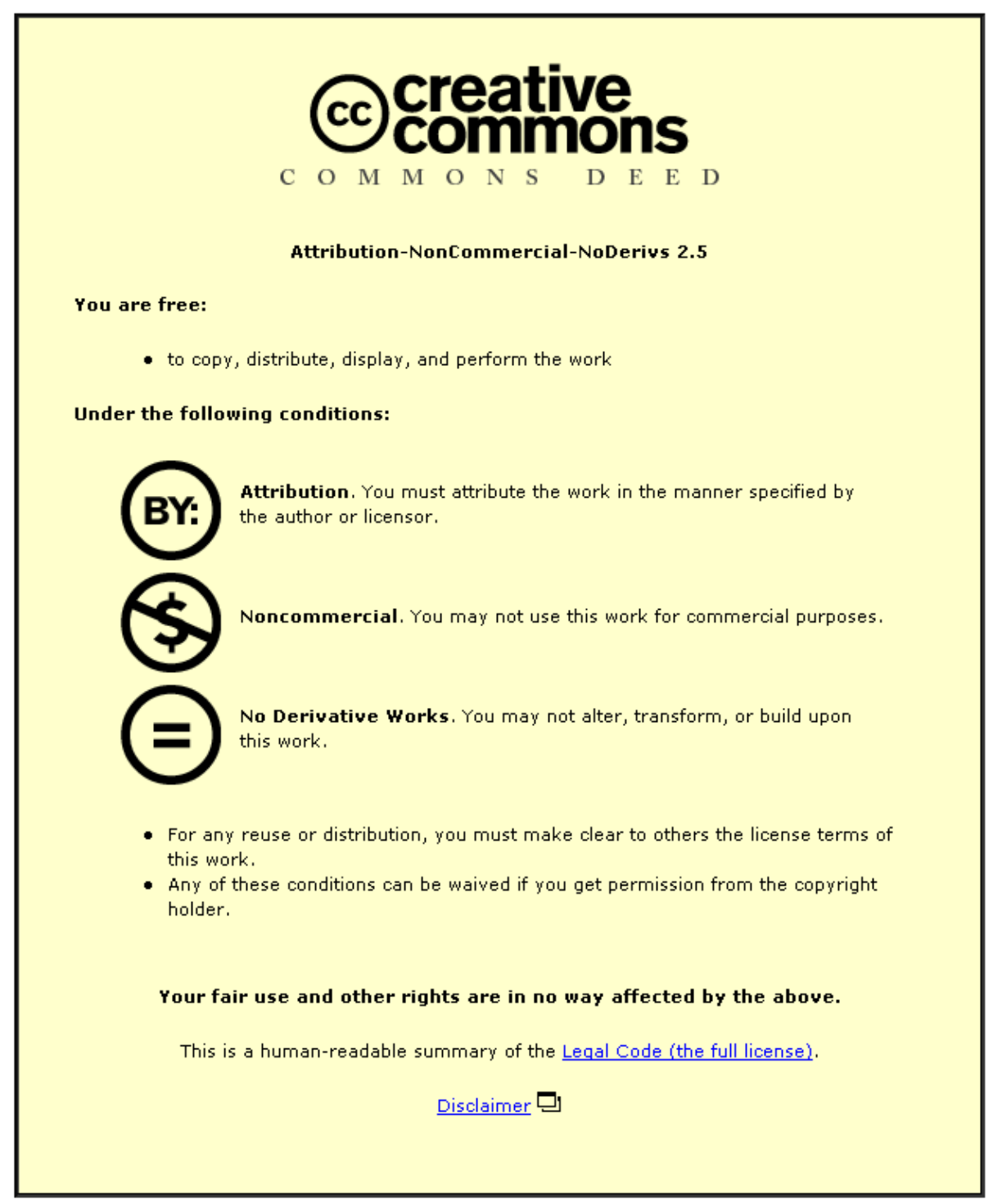

For the full text of this licence, please go to: http://creativecommons.org/licenses/by-nc-nd/2.5/ 


\title{
The Markov network fitness model
}

\author{
Alexander E.I. Brownlee, John A.W. McCall and Siddhartha K. Shakya
}

\begin{abstract}
Fitness modelling is an area of research which has recently received much interest among the evolutionary computing community. Fitness models can improve the efficiency of optimisation through direct sampling to generate new solutions, guiding of traditional genetic operators or as surrogates for a noisy or long-running fitness functions. In this chapter we discuss the application of Markov networks to fitness modelling of black-box functions within evolutionary computation, accompanied by discussion on the relationship between Markov networks and Walsh analysis of fitness functions. We review alternative fitness modelling and approximation techniques and draw comparisons with the Markov network approach. We discuss the applicability of Markov networks as fitness surrogates which may be used for constructing guided operators or more general hybrid algorithms. We conclude with some observations and issues which arise from work conducted in this area so far.
\end{abstract}

\section{Introduction, motivation and alternatives}

The end-goal of an evolutionary algorithm is usually efficient optimisation. Fitness modelling [20] is one of the many techniques which can support this goal, by reducing overall run-time or by simplifying the problem in some way.

Previous work including $[4,51,52,50]$ has described a number of approaches using undirected probabilistic graphical models (Markov networks) within a framework called Distribution Estimation Using Markov networks (DEUM), an Estimation of Distribution algorithm [24]. With the Markov network approach model-

\footnotetext{
A. Brownlee

Loughborough University, Loughborough, UK, e-mail: a.e.i.brownlee@lboro.ac.uk

J. McCall

Robert Gordon University, Aberdeen, UK e-mail: j.mccall@rgu.ac.uk

S. Shakya

BT Innovate \& Design, Ipswich, UK e-mail: sid.shakya@bt.com
} 
building overhead is significant, particularly as the number of variables and interactions increases. While the number of function evaluations to reach an optimum were significantly fewer than with other algorithms in our earlier work the time taken to build and sample the probabilistic model was large. However, it has been found that the Markov network at the heart of DEUM represents a good model of fitness [5, 7], and this has been exploited in recent work [8] as well as providing an explanation for the previous good results.

The broader concepts of fitness modelling, approximation and surrogates [20, 26] have attracted much interest among the evolutionary computing community. A motivation for using fitness modelling within an evolutionary algorithm is to improve the efficiency of optimisation; this can be achieved in several ways. If evaluating the model is cheaper than evaluating the fitness function, it can be used to reduce overall run-time [21, 41, 57, 62, 31]. A model may be used where no explicit fitness function exists such as in evolutionary art and music [22]. Further, a fitness model may be employed to simplify the search by reducing noise $[42,58,10]$ or smoothing a multimodal landscape [63].

Many approaches to fitness modelling exist. One common approach is artificial neural networks [21], with more recent examples including [57] and [14]. In [33] an algorithm groups individuals of similar fitness into classes that are then passed to Bayesian classifiers which can be sampled to generate individuals of high fitness. Schmidt and Lipson [48] use co-evolution to generate fitness predictors. In [12], an archive of already-evaluated solutions are fuzzily matched to new solutions, with fitness taken from the archive if matches are found. The Learnable Evolution Model (LEM) [32] incorporates machine learning to identify features distinguishing high and low fitness individuals. In [13], the authors report the use of a Gaussian random field meta-model as a surrogate in a $(\mu+\lambda)$ ES for single and multi-objective continuous problems with good results on a number of benchmarks.

Fitness inheritance (passing of fitness values from parents to offspring) to reduce the number of fitness evaluations $[11,56]$ is arguably a form of fitness modelling. A fitness model may also be used to guide standard genetic operators such as crossover and mutation as in $[27,1,21,40,60]$. Other hybrid approaches combine probabilistic models with different algorithms such as that described by [61, 36, 47]. In contrast to the use of undirected models in this chapter there are has also been some work done using directed probabilistic graphical models for fitness modelling, such as that described as a variant of fitness inheritance in [38].

Polynomial regression or the fitting of a response surface has also been used to construct a model of fitness [64]. The Markov fitness model described in this chapter bears some similarity to this, in effect being a response surface for the fitness function.

Much of the above work concentrates on continuous fitness functions. The Markov fitness model described here models discrete functions and there are a number of related works with such functions. One of the earliest is [35], where the authors use a neural network to classify low and high fitness solutions with a bit-string encoding. In [65], the authors use a meta-model built using machine learning techniques (one example is genetic programming), also to classify high and low fitness 
solutions having a bit-string encoding. Some approaches map the discrete function onto a continuous space: in [25], a radial basis function network is used as a surrogate for a mixed-integer search space. The model is used as a filter; too many offspring are created each generation and the model is used to choose promising ones which are evaluated with the true fitness function and retained. Similar to this, [59] proposes candidate over sampling in EBCOA [33]; generating too many solutions and trimming back to the number required. The authors found that picking the solutions predicted by the model to be less fit worked best. Their work looked at bit-string encoded functions and used machine learning approaches to infer rules distinguishing high and low fitness solutions.

Han and Yang [18] describe mapping discrete variables onto continuous ones to allow multiple linear regression for screening variables prior to optimisation. In [30], Gaussian processes are used for learning discrete fitness landscapes, demonstrated on Multidimensional Gaussian Landscapes and NK landscapes. Takahashi et al [58] use fitness estimation from a statistical model of the history of solutions to deal with noisy fitness functions; the example given is on a weight vector with discrete values. This builds on the earlier work with continuous functions in [42]. Finally, in [34] the author builds a surrogate from a Gibbs model which is derived from the distribution learnt by an EDA. This is demonstrated with both discrete and continuous benchmark functions.

Jin's comprehensive 2005 review [20] presents a wider survey of existing work in this area, and further recent developments can be found in [55].

\section{Defining the model}

Previous publications on DEUM including $[54,5]$ describe how a Markov network is used to model the distribution of energy across the set of variables in a bit-string encoded problem. In this section we summarise how the model is derived.

A Markov Network is a pair $(G, \Psi)$, where $G$ is the structure and the $\Psi$ is the parameter set of the network. $G$ is an undirected graph where each node corresponds to a random variable in the modelled data set and each edge corresponds to a probabilistic joint dependency between variables. We say that two nodes connected by an edge are neighbouring nodes. A subset $K=\left\{X_{i 1}, \ldots, X_{i k}\right\}$ of $k$ mutuallyneighbouring nodes is termed a $k$-clique. Note that we include the empty set $\varnothing$ as a 0 -clique and each singleton 1-clique $\left\{x_{i}\right\}$ in our definition of clique.

The Hammersley-Clifford Theorem states that the joint probability distribution of a Markov Network factorises as a Gibbs Distribution, completely determined by a set $\Psi$ of parameters, each of which is a real number $\alpha_{k}$ that defines the energy contribution from clique $k$.

The precise form of the Gibbs Distribution is given in equation (1):

$$
p(x)=\frac{e^{-U(x) / T}}{Z}
$$


where,

$$
Z=\sum_{y \in \Omega} e^{-U(y) / T}
$$

Here, the numerator, $e^{-U(x) / T}$, represents the likelihood of a particular configuration $x$ of the variables. The denominator, $Z$, is the normalising constant computed by summing over the set $\Omega$ of all possible configurations (note $Z$ is never computed in practice). $U(x)$ is an energy function computed by summing contributions calculated from the values that $x$ takes on each clique. Thus this exponentiated sum gives a factorisation of the distribution based on the structure $G$. We will consider the energy function in more detail shortly. $T$ is a temperature constant which controls the ruggedness of the probability distribution.

The key idea of the DEUM EDA is to model solution fitness as a mass distribution that equates to the Gibbs distribution as shown in equation (3):

$$
p(x)=\frac{f(x)}{\sum_{y \in \Omega} f(y)}=\frac{e^{-U(x) / T}}{Z}
$$

Sampling this distribution will generate high fitness individuals with high probability. We now explain how the DEUM algorithm estimates this distribution.

Identifying corresponding terms in the numerator and denominator gives, for each solution $x=\left\{x_{1}, x_{2}, \ldots, x_{n}\right\}$, the following negative log relationship between $f(x)$ and $U(x)$ :

$$
-\ln (f(x))=U(x)
$$

Let structure $G$ contain a set of cliques $K=\left\{K_{1}, \ldots, K_{m}\right\}$. Then, for any solution $x=\left\{x_{1}, x_{2}, \ldots, x_{n}\right\}, U(x)$ has the form:

$$
U(x)=\sum_{i} \alpha_{i} V_{K_{i}}(x)
$$

The $V_{k}$ are the characteristic functions of a Walsh decomposition of the fitness function. Walsh functions $[3,15,16,17]$ are a set of rectangular waveforms taking two amplitude values, +1 and -1 . Similar to the use of Fourier transforms representing for analogue waveforms, Walsh functions may be combined linearly to represent any fitness function based on a bit-string representation.

The $V_{K}(x)$ for each clique $K$ are defined in (6) to (8).

$$
\begin{gathered}
K=\varnothing \quad V_{\varnothing}(x) \equiv 1 \forall x \\
K=\{i\} \quad V_{i}(x)= \begin{cases}1 & x_{i}=1 \\
-1 & x_{i}=0\end{cases} \\
\text { For } K \subseteq|1, \ldots, n|, \quad|K| \geqslant 2, \quad V_{K}(x)=\prod_{i \in K} V_{i}(x)
\end{gathered}
$$


Thus the energy function, and hence the fitness, is completely determined by the parameters $\alpha_{i}$. The $\alpha_{i}$ are non-zero only for cliques present in the structure $G$. Given a sufficiently-sized population of solutions and their fitnesses, equations (4) and (5) yield a system of equations in the parameters that can be solved using a least-squares approximation to estimate the distribution. [50] describes how singular value decomposition [39] is used for this.

Of principle interest here is that, once the parameters are determined, we can combine (4) and (5) to obtain a model of the fitness function:

$$
-\ln (f(x))=\sum_{i} \alpha_{i} V_{K_{i}}(x)
$$

We call this the Markov Fitness Model (MFM) of $f$. We now proceed to discuss how the quality of the model may be measured before moving on to applications.

\subsection{Model quality and the fitness prediction correlation}

Given the relationship in (9), it is possible to extrapolate information about the fitness function and in particular the optimal solution by looking at the values given to the model parameters $\alpha_{i}$. Minimising the energy of a solution is equivalent to maximising fitness. For the univariate terms this means that a positive $\alpha_{i}$ will require a negative value for $V\left(x_{i}\right)$ to minimise the energy contribution from that term. This equates to $x_{i}$ (the $i$ th bit) being set to 0 . Likewise, a negative $\alpha_{i}$ value indicates the $i$ th bit should be set to 1 to minimise the contribution from that term. For bivariate terms, a positive $\alpha_{i j}$ value indicates that the two bits $x_{i}$ and $x_{j}$ associated with it should be opposite in value, to minimise the contribution from the term involving $V\left(x_{i j}\right)$. Similarly, a negative $\alpha_{i j}$ indicates that they should take the same value. This principle can be further extended to multivariate terms.

In [9] such analysis of MFM coefficients revealed a clear relationship with properties underlying the fitness function. This was despite having used a very small number (120) of function evaluations. Fitness was modelled for a bio-control problem, where bits set to 1 indicated times that nematode worms should be applied to a mushroom crop for control of the pest sciarid fly. The coefficients pointed towards application of the nematodes at points which matched the lifecycle of the fly larvae. Analysis of univariate and bivariate model coefficients for a number of further fitness functions was conducted in [4] and reinforced this finding.

The relationship between model coefficients and the global optimum is further illustrated here for the Checkerboard problem [2, 24]. The objective for this is to realise a grid with a checkerboard pattern of alternating $1 \mathrm{~s}$ and 0 s; each 1 should be surrounded by 0 s and vice versa, not including corners. Interactions occur between neighbours on the lattice without wrapping around at the edges. The 2D lattice structure for a 25-bit instance of the problem is illustrated in Figure 2. We constructed the MFM using the perfect structure for the problem (that is, univariate terms for each variable $X_{i}$ and bivariate terms for each neighbouring pair of variables $X_{i} X_{j}$ 


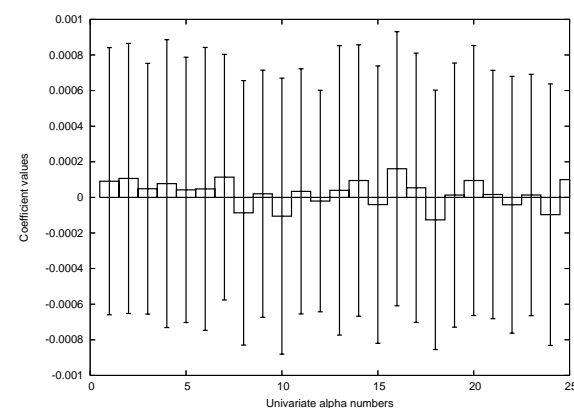

Fig. 1a Univariate alpha coefficients for 2D checkerboard

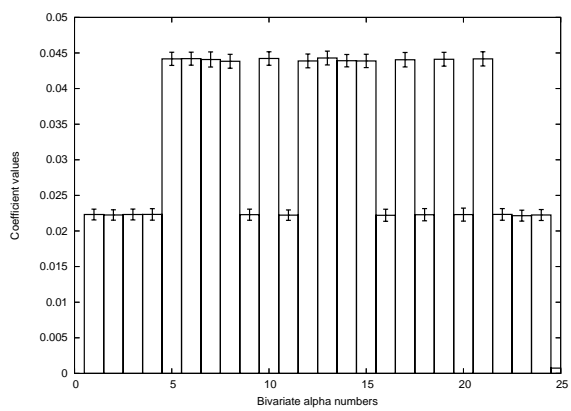

Fig. 1b Bivariate alpha coefficients for 2D checkerboard

on the lattice) and the model parameters were estimated using the fittest 220 solutions from a randomly generated starting population of 300 solutions. This process was repeated for 100 random starting populations, and the mean and standard deviation for each coefficient value over the 30 runs was computed. The coefficient values for the univariate and bivariate terms are illustrated in Figures 1a and 1b respectively. The univariate alphas are all close to zero: this is because there are two global optima which have complementary bits set to 1 and 0 , so the model does not bias individual variables towards one value. The bivariate alphas are all positive, indicating that neighbouring variables should be opposite in value. Of particular interest is that several of the bivariate alpha values are approximately double the magnitude of the others. The higher alpha values correspond to the interactions in the middle of the lattice (that is, neither of the variables they are associated with is on the edge of the checkerboard); these are dashed in Figure 2. These have a greater

Fig. 2 Model structure for 25 bit $2 \mathrm{D}$ Checkerboard Problem - numbers beside interactions correspond to those on the horizontal axis in Figure 1b. Note that the dashed interactions match the coefficients with a higher magnitude.

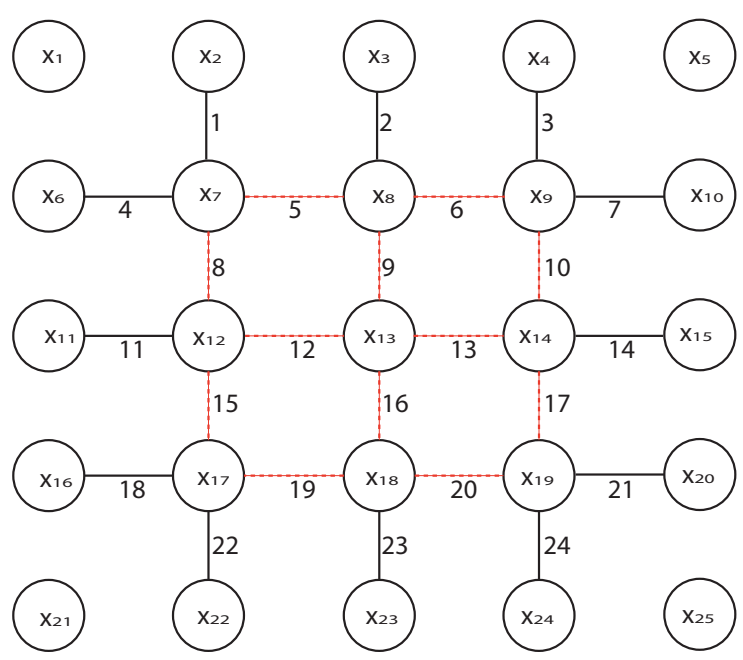




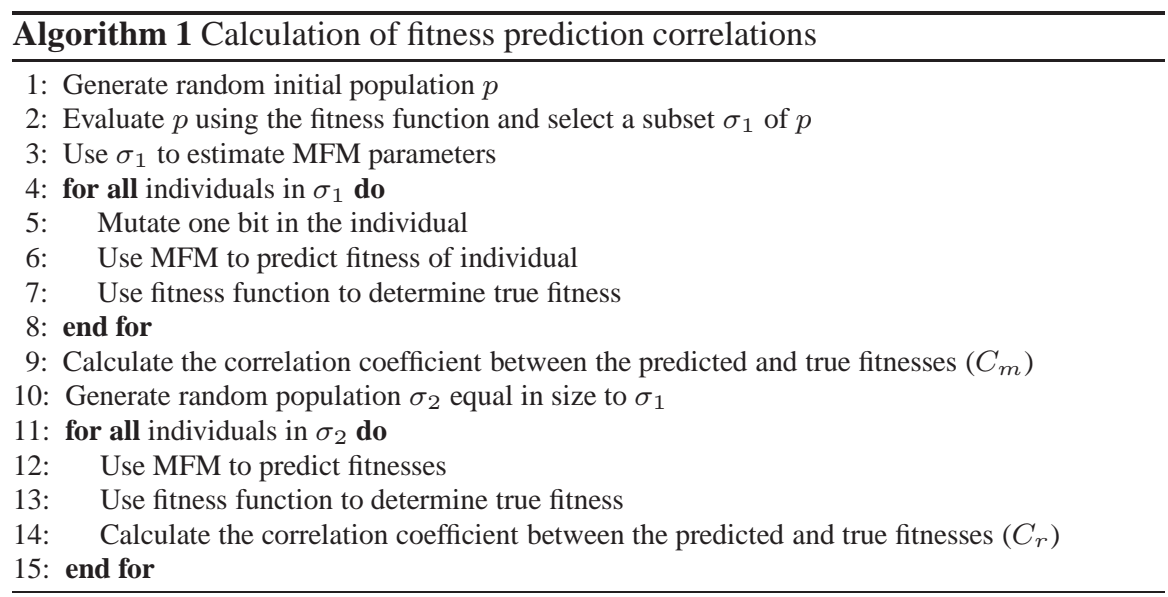

influence on fitness than those near the edge because if they break the constraint of neighbours not matching, their neighbours will also be affected. Thus we can see that the model places greater importance on these alphas. This shows that the MFM provides us with more information about the fitness function than simply pointing us in the direction of the global optimum.

A quantitative measure of model quality is also useful and the Fitness prediction correlation (FPC) [5] serves this purpose. This measures the MFM's ability to predict the fitness of unseen solutions. It is the Spearman's rank correlation [28] between the set of true fitnesses and fitnesses predicted by the model of an unseen population. Rank correlation is used because for discrete optimisation, it is only necessary to rank individuals in order of relative fitness. Predicting fitness is simply a reversal of the process used to estimate the $\alpha_{K}$; the variable values for a solution being substituted into (9).

[7] defines two variants of FPC $-C_{m}$ and $C_{r}$. $C_{r}$ measures fitness prediction capability for randomly generated individuals; it follows that if this high then the MFM closely models the general fitness function. $C_{m}$ measures fitness prediction capability for solutions neighbouring the current population, which is important for using the model to guide operators such as mutation. $C_{m}$ and $C_{r}$ are calculated by following Algorithm 1.

Given the right conditions (particularly when the model structure closely matches the non-zero components of the Walsh expansion of the fitness function, and large population), FPC values for the MFM can be close to $1[5,7,6,4]$. This indicates a strong correlation between predicted and true fitnesses for complex problems such as Ising and MAXSAT. This explains the success of the optimisation approaches using the MFM described in the next section. 


\section{Applications}

There are several ways to exploit the model of fitness in the MFM. Within DEUM, direct sampling is used to generate new solutions with a high probability of being high in fitness $[54,53,5]$. This direct sampling of the fitness model rather than the fitness function has the benefit that the model can make the problem easier for the search part of the algorithm - the smoothing effect described in [63]. For example, in [54], it was found that directly sampling solutions from the MFM rather than the true fitness function for 2D Ising increased the success rate of a bitwise Gibbs sampler from $87 \%$ to $99 \%$. It was concluded that by using a real-valued search space within the MFM rather than the discrete values within the fitness function, the fitness landscape was altered to allow the algorithm to escape being trapped within plateaux, leading to more efficient searching of the landscape.

\subsection{MFM-GA}

[8] proposes MFM-GA, applying the MFM as a surrogate in a genetic algorithm. The model is constructed at the start of the run, then the GA samples evaluations exclusively from the model. A number of benchmark functions were used as a proofof-concept, then the algorithm was applied to a computationally expensive fitness function - feature selection for case-based reasoning. Promising results were reported, with a significant reduction in overall run-time over a GA. A major issue with the approach is that the model is only constructed once, making MFM-GA sensitive to modelling errors, with the result that the optimisation could not find solutions as fit as the GA. However, the solutions found were fitter than those found using a CBR-specific optimiser and represent something of a compromise between finding the fittest solution and a short run time.

\subsection{Guided local search}

A previously unpublished approach is to use the MFM as a fitness surrogate for a local search; allowing the local search many iterations without consuming many fitness evaluations. The MFM is used to filter solutions for evaluation by the true fitness function; this is similar to the approaches in [25, 13, 59].

This was applied with some success to the Huygens probe problem [29], part of a competition at the 2006 Congress on Evolutionary Computation (CEC). The objective of the problem is to find the lowest point on each of a series of 20 "moons" - fractal landscapes that are wrapped in both $\mathrm{x}$ and $\mathrm{y}$ dimensions. For each moon an algorithm is restricted to 1000 probes (fitness evaluations).

A bit-string encoding of the coordinate values was used. The algorithm rebuilds the model around progressively smaller areas of the lunar surface, suited to the frac- 
tal nature of the landscape (equal levels of detail at different zoom levels). Only univariate terms were included in the model; given that it is looking at neighbouring solutions to those used to build the model (those a short Hamming distance away), the results in Chapter 4 of [4] indicate that this should be enough to provide a reasonable fitness prediction capability. The workflow of the guided local search for this problem is given in Algorithm 2.

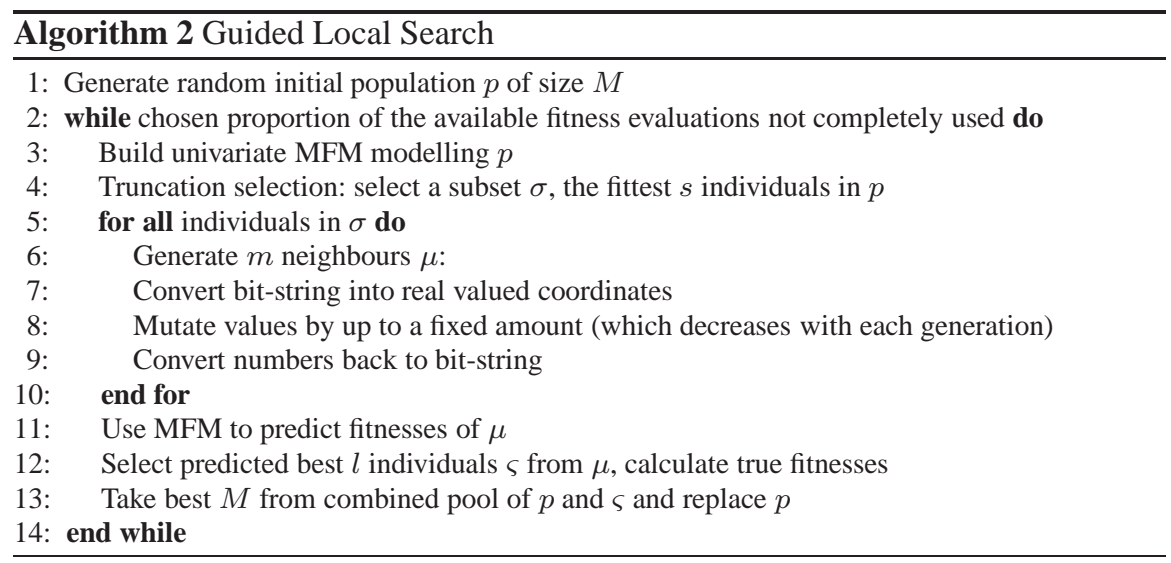

Once the algorithm terminated with a single best solution $x$, the remainder of the 1000 evaluations were used for an exhaustive search of the neighbours to $x$. The proportion of evaluations allocated to each stage fixed per run; in the first instance the guided local search was given $2 / 3$ of the total, in the second it was given 3/4.

Results for this algorithm were compared with the others taking part in the CEC competition. Each algorithm was run on the same set of 100 randomly generated moons, with a central server providing fitness evaluations and performing comparisons between algorithms via a SOAP interface. The algorithm performed comparably with a number of well-known problem solvers such as evolutionary strategies (ES), memetic algorithms (MA) and simulated annealing (SA), coming in 9th and 11 th out of 16 with the two configurations. Unfortunately no more data is available on the specific implementations of these algorithms; however, these results do show that the approach is competitive with a wide range of others on this black-box problem.

\section{Issues affecting modelling capability}

There are a number of factors which affect the quality of the fitness model, which also provide the grounding for future study. 


\subsection{Model building time}

Of particular note is that the singular value decomposition used to estimate model parameters is $O\left(N^{2} \mathrm{~m}\right)$ complexity in the number of model coefficients $N$ and the population size $m$, meaning that building the model becomes expensive for increasing problem sizes and complexities. In much of our work, the population has had to be large enough to be slightly over-specified $(m>N)$, so the overall model building complexity is $O\left(N^{3}\right)$. This is comparable to other EDAs - for example hBOA is dominated by Bayesian network structure learning complexity of $O\left(k n^{2} m\right)$ [37], for problems of $n$ variables which can be decomposed into subproblems of order $k$. Note however that for the MFM, the number of model coefficients $N$ typically includes terms for each problem variable and for each interaction, so $N$ is usually greater than the number of variables $n$ in the problem, particularly for problems with many high-order interactions. A method of building the model incrementally or with multiple threads would help to mitigate this issue.

\subsection{Model structure}

The structure of the MFM strongly influences its fitness modelling capability; in $[7,4]$ we observed the impact on fitness prediction capability of removing different cliques from the model. It is known that not all interactions which are present in a problem will necessarily be required in the model for the algorithm to rank individuals by fitness and find a global optimum. This observation is related to the concepts of necessary and unnecessary interactions [19] and benign and malign interactions [23]. This is also related to spurious correlations [45], false relationships in the model resulting from selection. Much of our previous work with the MFM has used a fixed structure derived from the problem definition, but for black-box problems in particular, the structure is unknown and must be inferred by sampling the fitness function. Works describing structure learning techniques for Markov networks include [43, 44, 46, 49]; the issue of structure learning specifically for the MFM in DEUM is explored in [6] and [51]. Approaches have typically involved conducting dependency tests such as Chi-Square on pairs of variables, either using existing members of the population or generating new solutions by mutating specific variables. A deterministic clique-finding algorithm can then be run on the resulting graph to find higher order cliques.

We further explore the issue of model and problem structure in relation to fitness modelling in [7, 4]. There we introduce the terms perfect and imperfect; perfect referring to the ideal structure with exactly the same interactions as present in the fitness function and imperfect referring any other structure. 


\subsection{Population size and selection}

There is a clear and quantifiable relationship between the number of solutions present in the population used to estimate model parameters and the fitness modelling capability. In [7] and more extensively in [4], a number of experiments show the effect of structure, population size and selection on the fitness prediction correlation. As population size reaches, then exceeds, the number of parameters in the model, there is a sharp transition from near zero to strong positive correlation between model and fitness function, for a number of fitness functions including onemax, Ising, MAXSAT and Trap-k. An example is given in Figure 3. With imperfect structures the model can typically predict fitnesses of solutions neighbouring those in the training population, but not those of randomly generated solutions.

The MFM is a probabilistic distribution over the fitness function, so sampling the model produces solutions with a high probability of being high in fitness. This means that explicit selection is not required for building the model; however selection still plays a crucial role in improving the fitness modelling capability [7, 4]. This is particularly the case where the population is too small or the model structure is imperfect, where selection sharpens the information about fitness already present in the population.

\section{Conclusion and future work}

In this chapter we have explained how the MFM applies Markov networks to fitness modelling of discrete problems. This may be exploited by directly sampling the model to generate new solutions, or by using the model as a surrogate, predicting fitness of solutions and filtering out promising ones for evaluation. Population size, model structure and selection are important factors in establishing a good model of fitness. Further exploration of these factors will help us to build better and more

Fig. 3 FPC with increasing population size for 1000 bit onemax. Note the rapid increase in both FPC figures at the point where the population size exceeds 1001; the number of coefficients in the model for this problem (1000 univariate terms plus the constant).

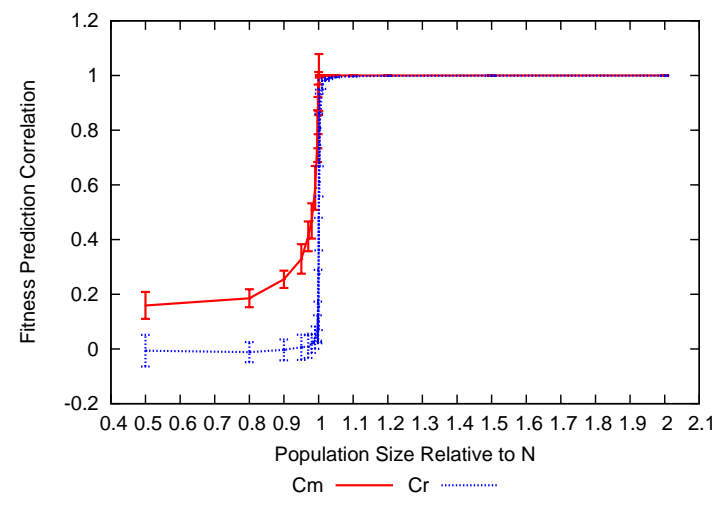


useful models of fitness, and also provide better understanding of how fitness is distributed within a population, which will be of use to the wider EA community. Further, the MFM is currently limited to fitness functions having a binary representation. Extension to higher cardinality or continuous representations would result in a more generally applicable model of fitness; this could entail adding additional terms to the model for each possible value. Finally, improving the efficiency of the model building process will increase the number of situations where it offers an overall performance improvement.

\section{References}

1. Abboud, K., Schoenauer, M.: Surrogate Deterministic Mutation: Preliminary Results. In: Selected Papers from the 5th European Conference on Artificial Evolution, pp. 104-116. Springer-Verlag, London, UK (2002)

2. Baluja, S., Davies, S.: Using optimal dependency-trees for combinational optimization. In: ICML '97: Proceedings of the Fourteenth International Conference on Machine Learning, pp. 30-38. Morgan Kaufmann Publishers Inc (1997)

3. Bethke, A.: Genetic Algorithms as Function Optimizers. Ph.D. thesis, University of Mitchigan (1980)

4. Brownlee, A.E.I.: Multivariate Markov Networks for Fitness Modelling in an Estimation of Distribution Algorithm. Ph.D. thesis, Robert Gordon University, Aberdeen (2009). URL http://hdl.handle.net/10059/381

5. Brownlee, A.E.I., McCall, J.A.W., Brown, D.F.: Solving the MAXSAT problem using a multivariate EDA based on Markov networks. In: Proceedings of the Genetic and Evolutionary Computation COnference (GECCO 2007) (Late Breaking Papers), pp. 2423-2428. ACM Press, New York, NY, USA (2007)

6. Brownlee, A.E.I., McCall, J.A.W., Shakya, S.K., Zhang, Q.: Structure Learning and Optimisation in a Markov-network based Estimation of Distribution Algorithm. In: Proceedings of the IEEE Congress on Evolutionary Computation (CEC 2009), CEC'09, pp. 447-454. IEEE Press, Trondheim, Norway (2009)

7. Brownlee, A.E.I., McCall, J.A.W., Zhang, Q., Brown, D.: Approaches to Selection and their effect on Fitness Modeling in an Estimation of Distribution Algorithm. In: Proceedings of the IEEE World Congress on Computational Intelligence (CEC 2008), pp. 2621 -2628. IEEE Press, Hong Kong, China (2008)

8. Brownlee, A.E.I., Regnier-Coudert, O., McCall, J.A.W., Massie, S.: Using a Markov network as a surrogate fitness function in a genetic algorithm. In: Proceedings of the IEEE Congress on Evolutionary Computation (CEC 2010), pp. 4525-4532. IEEE Press, Barcelona, Spain (2010)

9. Brownlee, A.E.I., Wu, Y., McCall, J.A.W., Godley, P.M., Cairns, D.E., Cowie, J.: Optimisation and fitness modelling of bio-control in mushroom farming using a Markov network EDA. In: Proceedings of the Genetic and Evolutionary Computation COnference (GECCO 2008), pp. 465-466 (2008). Atlanta, Georgia, USA, ACM.

10. Bui, L.T., Abbass, H.A., Essam, D.: Fitness inheritance for noisy evolutionary multi-objective optimization. In: Proceedings of the 2005 conference on Genetic and evolutionary computation, GECCO '05, pp. 779-785. ACM, New York, NY, USA (2005)

11. Chen, J.H., Goldberg, D., S.-Y.Ho, K.Sastry: Fitness inheritance in multiobjective optimization. In: Proceedings of the Genetic and Evolutionary Computation COnference (GECCO 2002), pp. 319-326. ACM Press (2002)

12. Davarynejad, M., Ahn, C.W., Vrancken, J., van den Berg, J., Coello Coello, C.A.: Evolutionary hidden information detection by granulation-based fitness approximation. Appl. Soft Comput. 10, 719-729 (2010) 
13. Emmerich, M., Giannakoglou, K., Naujoks, B.: Single- and multiobjective evolutionary optimization assisted by gaussian random field metamodels. Evolutionary Computation, IEEE Transactions on 10(4), $421-439$ (2006)

14. Furtuna, R., Curteanu, S., Leon, F.: An elitist non-dominated sorting genetic algorithm enhanced with a neural network applied to the multi-objective optimization of a polysiloxane synthesis process. Eng. Appl. Artif. Intell. 24, 772-785 (2011)

15. Goldberg, D.: Genetic Algorithms and Walsh Functions: Part I, A Gentle Introduction. Complex Systems 3, 129-152 (1989)

16. Goldberg, D.: Genetic Algorithms and Walsh Functions: Part II, Deception and its Analysis. Complex Systems 3, 153-171 (1989)

17. Golubov, B., Efimov, A., Skvortsov, V.: Walsh Series and Transforms: Theory and Applications, Mathematics and Applications: Soviet Series, vol. 64. Kluwer Academic Publishers, Boston (1991)

18. Han, S.H., Yang, H.: Screening important design variables for building a usability model: genetic algorithm-based partial least-squares approach. International Journal of Industrial Ergonomics 33(2), 159 - 171 (2004)

19. Hauschild, M., Pelikan, M., Lima, C.F., Sastry, K.: Analyzing probabilistic models in hierarchical BOA on traps and spin glasses. In: Proceedings of the Genetic and Evolutionary Computation COnference (GECCO 2007), pp. 523-530. ACM Press, New York, NY, USA (2007)

20. Jin, Y.: A comprehensive survey of fitness approximation in evolutionary computation. Soft Computing 9(1), 3-12 (2005)

21. Jin, Y., Sendhoff, B.: Reducing fitness evaluations using clustering techniques and neural network ensembles. In: Proceedings of the Genetic and Evolutionary Computation COnference (GECCO 2004), pp. 688-699. Springer, Seattle, WA (2004)

22. Johanson, B.: Gp-music: An interactive genetic programming system for music generation with automated fitness raters. In: Proceedings of the Third Annual Conference, pp. 181-186. MIT Press (1998)

23. Kallel, L., Naudts, B., Reeves, R.: Properties of fitness functions and search landscapes. In: L. Kallel, B. Naudts, A. Rogers (eds.) Theoretical Aspects of Evolutionary Computing, pp. 177-208. Springer Verlag (2000)

24. Larrañaga, P., Lozano, J.A.: Estimation of Distribution Algorithms: A New Tool for Evolutionary Computation. Kluwer Academic Publishers, Boston (2002)

25. Li, R., Emmerich, M., Eggermont, J., Bovenkamp, E., Back, T., Dijkstra, J., Reiber, J.: Metamodel-assisted mixed integer evolution strategies and their application to intravascular ultrasound image analysis. In: Evolutionary Computation, 2008. CEC 2008. (IEEE World Congress on Computational Intelligence). IEEE Congress on, pp. 2764 -2771 (2008)

26. Lim, D., Jin, Y., Ong, Y.S., Sendhoff, B.: Generalizing surrogate-assisted evolutionary computation. Evolutionary Computation, IEEE Transactions on 14(3), 329 -355 (2010)

27. Lima, C.F., Sastry, K., Goldberg, D.E., Lobo, F.G.: Combining competent crossover and mutation operators: a probabilistic model building approach. In: Proceedings of the 2005 conference on Genetic and evolutionary computation (GECCO 2005), pp. 735-742. ACM, New York, NY, USA (2005)

28. Lucey, T.: Quantatitive Techniques: An Instructional Manual. D. P. Publications, Eastleigh, Hampshire, UK (1984)

29. MacNish, C.: Benchmarking Evolutionary Algorithms: The Huygens Suite. In: Proceedings of the Genetic and Evolutionary Computation COnference (GECCO 2005) (Late Breaking Papers), pp. 2423-2428. ACM Press, New York, NY, USA (2005)

30. Macready, W., Levitan, B.: Learning landscapes: regression on discrete spaces. In: Proceedings of the IEEE Congress on Evolutionary Computation (CEC 1999), vol. 1, pp. 687-694 (1999)

31. Magnier, L., Haghighat, F.: Multiobjective optimization of building design using trnsys simulations, genetic algorithm, and artificial neural network. Building and Environment 45(3), 739-746 (2010) 
32. Michalski, R.S.: Learnable evolution model: Evolutionary processes guided by machine learning. Machine Learning 38(1-2), 9-40 (2000)

33. Miquélez, T., Bengoetxea, E., Larrañaga, P.: Evolutionary computation based on Bayesian classifiers. International Journal of Applied Mathematics and Computer Science 14(3), 101115 (2004)

34. Ochoa, A.: Opportunities for expensive optimization with estimation of distribution algorithms. In: L.M. Hiot, Y.S. Ong, Y. Tenne, C.K. Goh (eds.) Computational Intelligence in Expensive Optimization Problems, Adaptation, Learning, and Optimization, vol. 2, pp. 193218. Springer Berlin Heidelberg (2010)

35. Ochoa, A.A., Soto, M.R.: Partial evaluation in genetic algorithms. In: Proceedings of the 10th international conference on Industrial and Engineering Applications of Artificial Intelligence and Expert Systems, IEA/AIE'1997, pp. 217-222. Goose Pond Press (1997)

36. Peña, J., Robles, V., Larrañaga, P., Herves, V., Rosales, F., Pérez, M.: GA-EDA: Hybrid evolutionary algorithm using genetic and estimation of distribution algorithms. In: IEA/AIE'2004: Proceedings of the 17th International Conference on Innovations in Applied Artificial Intelligence, Lecture Notes In Computer Science, pp. 361-371. Springer-Verlag (2004)

37. Pelikan, M.: Hierarchical Bayesian Optimization Algorithm - Toward a New Generation of Evolutionary Algorithms, Studies in Fuzziness and Soft Computing, vol. 170. Springer (2005)

38. Pelikan, M., Sastry, K.: Fitness inheritance in the Bayesian optimization algorithm. In: Proceedings of the Genetic and Evolutionary Computation COnference (GECCO 2004), pp. 4859 (2004)

39. Press, W.H., Flannery, B.P., Teukolsky, S.A., Vetterling, W.T.: Numerical Recipes: The Art of Scientific Computing. Cambridge University Press, Cambridge, UK (1986)

40. Rasheed, K., Vattam, S., Ni, X.: Comparison of methods for using reduced models to speed up design optimization. In: Proceedings of the Genetic and Evolutionary Computation COnference (GECCO 2002), pp. 1180-1187. Morgan Kaufmann Publishers Inc., San Francisco, CA, USA (2002)

41. Regis, R., Shoemaker, C.: Local function approximation in evolutionary algorithms for the optimization of costly functions. IEEE Transactions on Evolutionary Computation 8(5), 490 -505 (2004)

42. Sano, Y., Kita, H.: Optimization of noisy fitness functions by means of genetic algorithms using history of search with test of estimation. Computational Intelligence, Proceedings of the World on Congress on 1, 360-365 (2002)

43. Santana, R.: A Markov network based factorized distribution algorithm for optimization. In: Proceedings of the 14th European Conference on Machine Learning; Lecture Notes in Artificial Intelligence, vol. 2837, pp. 337-348. Springer-Verlag, Berlin (2003)

44. Santana, R., Larrañaga, P., Lozano, J.A.: Mixtures of Kikuchi Approximations. In: J. Fürnkranz, T. Scheffer, M. Spiliopoulou (eds.) Proceedings of the 17th European Conference on Machine Learning: ECML 2006, Lecture Notes in Computer Science, vol. 4212, pp. 365-376 (2006)

45. Santana, R., Larrañaga, P., Lozano, J.A.: Research topics on discrete estimation of distribution algorithms. Memetic Computing 1(1), 35-54 (2009)

46. Santana, R., Ochoa, A., Soto, M.R.: The mixture of trees factorized distribution algorithm. In: L. Spector, E. Goodman, A. Wu, W.B. Langdon, H.M. Voigt, M. Gen, S. Sen, M. Dorigo, S. Pezeshk, M. Garzon, E. Burke (eds.) Proceedings of the Genetic and Evolutionary Computation COnference (GECCO 2001), pp. 543-550. Morgan Kaufmann Publishers (2001)

47. Sastry, K., Lima, C., Goldberg, D.E.: Evaluation relaxation using substructural information and linear estimation. In: Proceedings of the Genetic and Evolutionary Computation COnference (GECCO 2006), pp. 419-426. ACM Press, New York, NY, USA (2006)

48. Schmidt, M.D., Lipson, H.: Coevolution of fitness predictors. IEEE Transactions on Evolutionary Computation 12(6), 736-749 (2008)

49. Shakya, S., Santana, R.: A Markovianity based optimisation algorithm. Tech. Rep. EHUKZAA-IK-3/08, Department of Computer Science and Artificial Intelligence, University of the Basque Country (2008) 
50. Shakya, S.K.: DEUM: A framework for an estimation of distribution algorithm based on Markov random fields. Ph.D. thesis, The Robert Gordon University, Aberdeen, UK (2006). URL http://hdl.handle.net/10059/39

51. Shakya, S.K., Brownlee, A.E.I., McCall, J.A.W., Fournier, F., Owusu, G.: A fully multivariate DEUM algorithm. In: Proceedings of the IEEE Congress on Evolutionary Computation (CEC 2009), pp. 479-486. IEEE Press (2009)

52. Shakya, S.K., McCall, J.A.W.: Optimization by estimation of distribution with DEUM framework based on Markov random fields. International Journal of Automation and Computing 4(3), 262-272 (2007)

53. Shakya, S.K., McCall, J.A.W., Brown, D.F.: Incorporating a Metropolis method in a distribution estimation using Markov random field algorithm. In: Proceedings of the IEEE Congress on Evolutionary Computation (CEC 2005), pp. 2576-2583. IEEE Press (2005)

54. Shakya, S.K., McCall, J.A.W., Brown, D.F.: Solving the Ising spin glass problem using a bivariate EDA based on Markov random fields. In: Proceedings of the IEEE World Congress on Computational Intelligence (CEC 2006). IEEE Press (2006)

55. Shi, L., Rasheed, K.: A Survey of Fitness Approximation Methods Applied in Evolutionary Algorithms. In: Y. Tenne, C.K. Goh (eds.) Computational Intelligence in Expensive Optimization Problems, Adaptation Learning and Optimization, vol. 2, chap. 1, pp. 3-28. Springer Berlin Heidelberg, Berlin, Heidelberg (2010)

56. Smith, R.E., Dike, B.A., Stegmann, S.A.: Fitness inheritance in genetic algorithms. In: SAC '95: Proceedings of the 1995 ACM symposium on Applied computing, pp. 345-350. ACM Press, New York, NY, USA (1995)

57. Syberfeldt, A., Grimm1, H., Ng, A., John, R.I.: A parallel surrogate-assisted multi-objective evolutionary algorithm for computationally expensive optimization problems. In: J. Wang. (ed.) Proceedings of the IEEE World Congress on Computational Intelligence (CEC 2008), pp. 3177-3184. IEEE Computational Intelligence Society, IEEE Press, Hong Kong (2008)

58. Takahashi, S., Kita, H., Suzuki, H., Sudo, T., Markon, S.: Simulation-based optimization of a controller for multi-car elevators using a genetic algorithm for noisy fitness function. In: Proceedings of the IEEE Congress on Evolutionary Computation (CEC 2003), vol. 3, pp. $1582-1587(2003)$

59. Wallin, D., Ryan, C.: Using over-sampling in a Bayesian classifier EDA to solve deceptive and hierarchical problems. In: Proceedings of the IEEE Congress on Evolutionary Computation (CEC 2009), pp. 1660 -1667 (2009)

60. Zhang, Q., Sun, J.: Iterated local search with guided mutation. In: Proceedings of the IEEE World Congress on Computational Intelligence (CEC 2006), pp. 924 - 929. IEEE Press (2006)

61. Zhang, Q., Sun, J., Tsang, E.: Combinations of estimation of distribution algorithms and other techniques. International Journal of Automation \& Computing pp. 273-280 (2007)

62. Zhou, L., Haghighat, F.: Optimization of ventilation system design and operation in office environment, part i: Methodology. Building and Environment 44(4), 651-656 (2009)

63. Zhou, Z., Ong, Y.S., Lim, M.H., Lee, B.S.: Memetic algorithm using multi-surrogates for computationally expensive optimization problems. Soft Comput. 11(10), 957-971 (2007)

64. Zhou, Z., Ong, Y.S., Nguyen, M.H., Lim, D.: A study on polynomial regression and Gaussian process global surrogate model in hierarchical surrogate-assisted evolutionary algorithm. In: Proceedings of the IEEE Congress on Evolutionary Computation (CEC 2005), vol. 3, pp. 2832-2839 (2005)

65. Ziegler, J., Banzhaf, W.: Decreasing the number of evaluations in evolutionary algorithms by using a meta-model of the fitness function. In: in Genetic Programming, Proceedings of EuroGP2003, pp. 264-275. Springer-Verlag (2003) 